\title{
Copper(I) Complexes of Pyridine-Bridged Phosphaalkene-Oxazoline Pincer Ligands
}

\author{
Spencer C. Serin, Fraser S. Pick, Gregory R. Dake,* Derek P. Gates* \\ Department of Chemistry, University of British Columbia, 2036 Main Mall, \\ Vancouver, British Columbia, Canada \\ E-mail:gdake@chem.ubc.ca,dgates@chem.ubc.ca
}

\section{Contents}

Figure S1 $\quad{ }^{1} \mathrm{H}^{-1} \mathrm{H}$ COSY and NOESY NMR Spectra of 1a $\quad$ S2

$\begin{array}{lll}\text { Figure S2 } & { }^{1} \mathrm{H}_{-}{ }^{31} \mathrm{P} \text { HMBC NMR spectrum of 1a } & \mathrm{S} 3\end{array}$

$\begin{array}{lll}\text { Figure S3 } & { }^{1} \mathrm{H}-{ }^{1} \mathrm{H} \text { NOESY NMR spectrum of complex } 7 & \mathrm{~S} 3\end{array}$

$\begin{array}{lll}\text { Figure S4 Optimized structure of 8a } & \text { S4 }\end{array}$

$\begin{array}{lll}\text { Figure S5 Optimized structure of 8d } & \text { S4 }\end{array}$

Table S1 Cartesian coordinates for optimized structure 8a $\quad$ S5

Table S2 Cartesian coordinates for optimized structure 8a $\quad$ S7

$\begin{array}{lll}\text { Table S3 Computed Low Energy Absorptions for 8a } & \text { S9 }\end{array}$

Table S4 Computed Low Energy Absorptions for 8d $\quad$ S9

Table S5 X-ray Data Collection and Refinement Details $\quad$ S10 

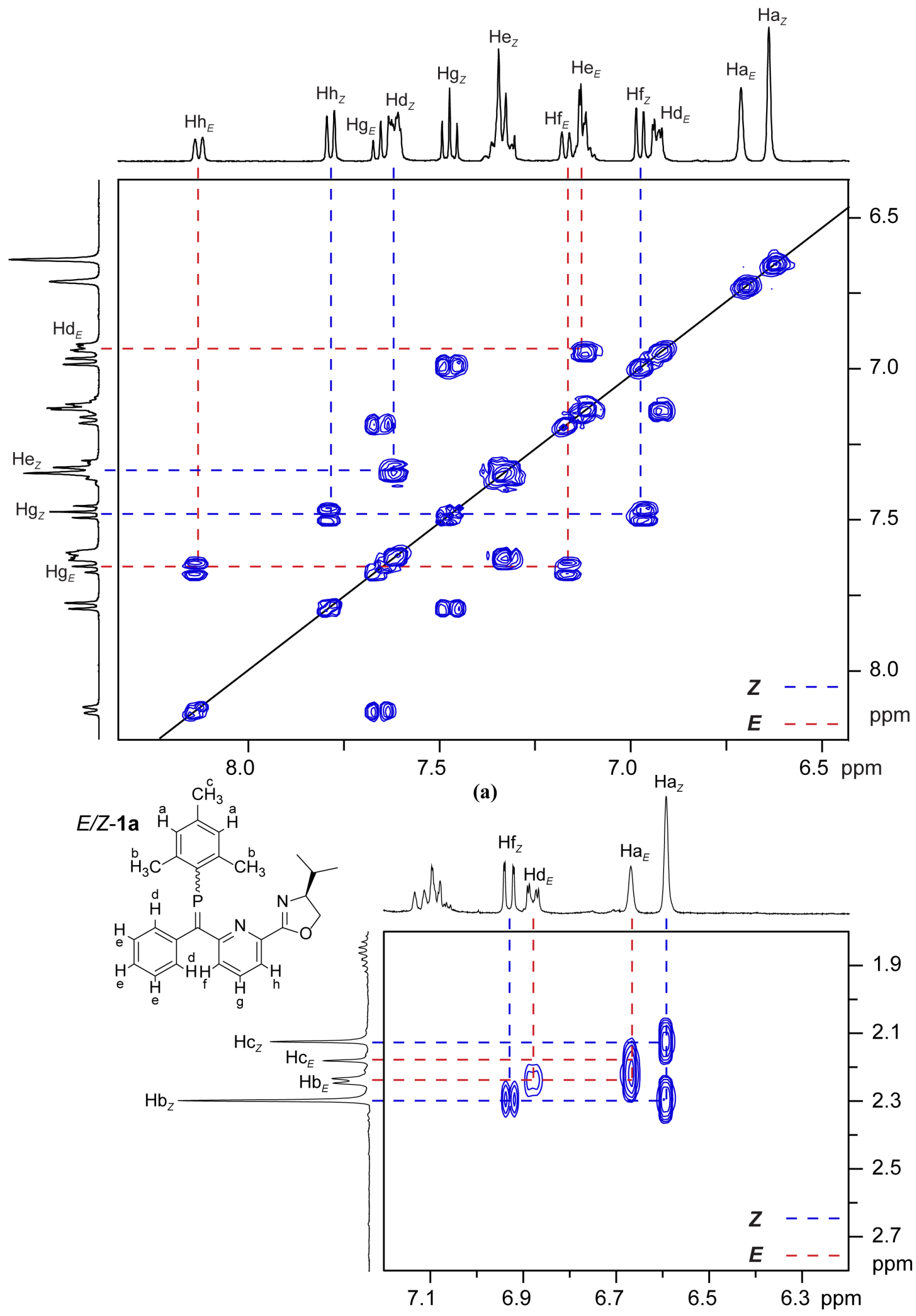

(b)

Figure S1 ${ }^{1} \mathrm{H}-{ }^{1} \mathrm{H}$ COSY (a) and NOESY (b) NMR Spectra (400 MHz, $298 \mathrm{~K}$ ) in $\mathrm{CDCl}_{3}$ of $E / Z-1 \mathbf{a}$. 


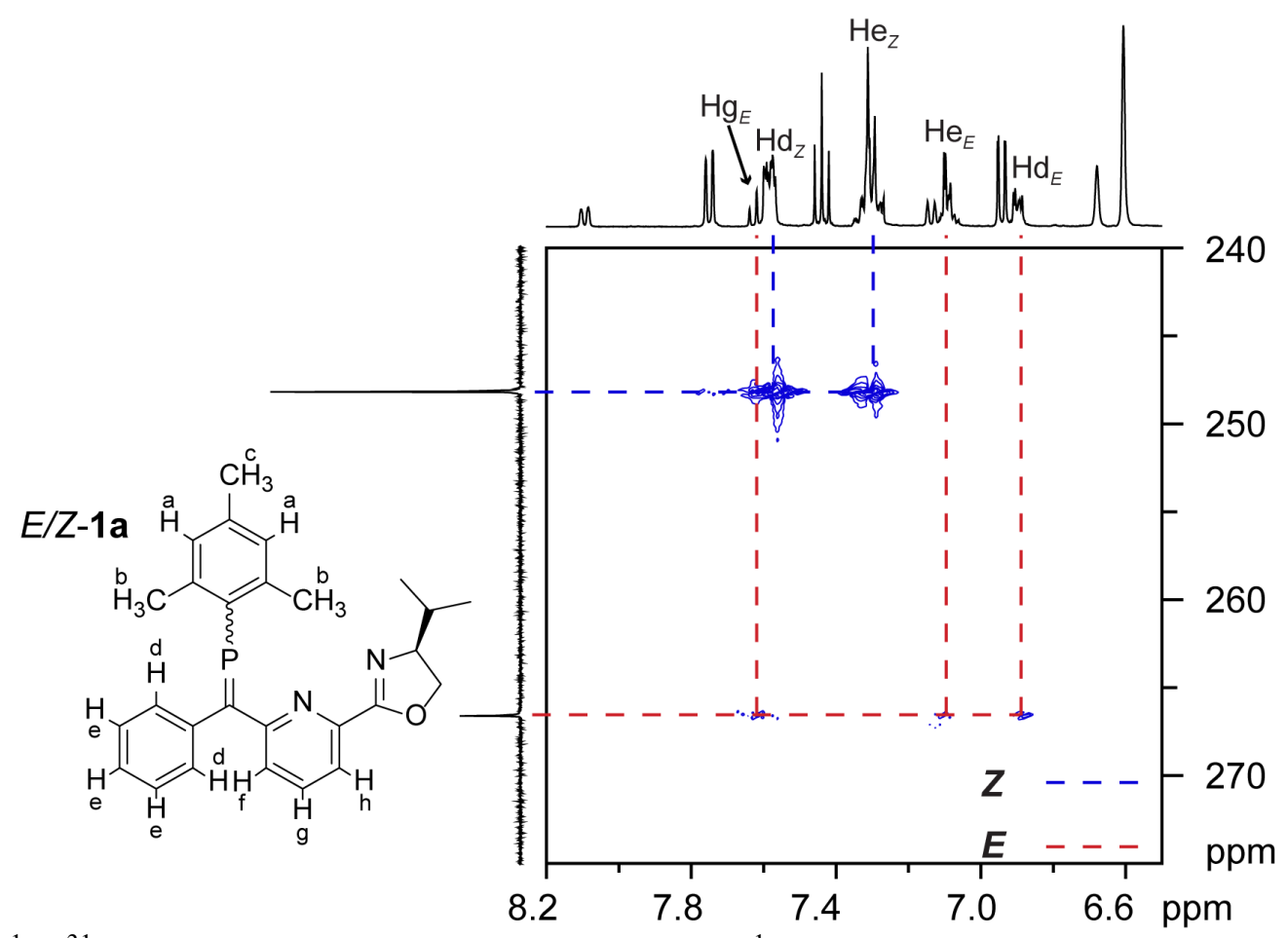

Figure S2 ${ }^{1} \mathrm{H}^{31} \mathrm{P}$ HMBC NMR spectrum $\left(400 \mathrm{MHz}\right.$ for $\left.{ }^{1} \mathrm{H}, 298 \mathrm{~K}\right)$ in $\mathrm{CDCl}_{3}$ of $E / Z-1 \mathbf{a}$.

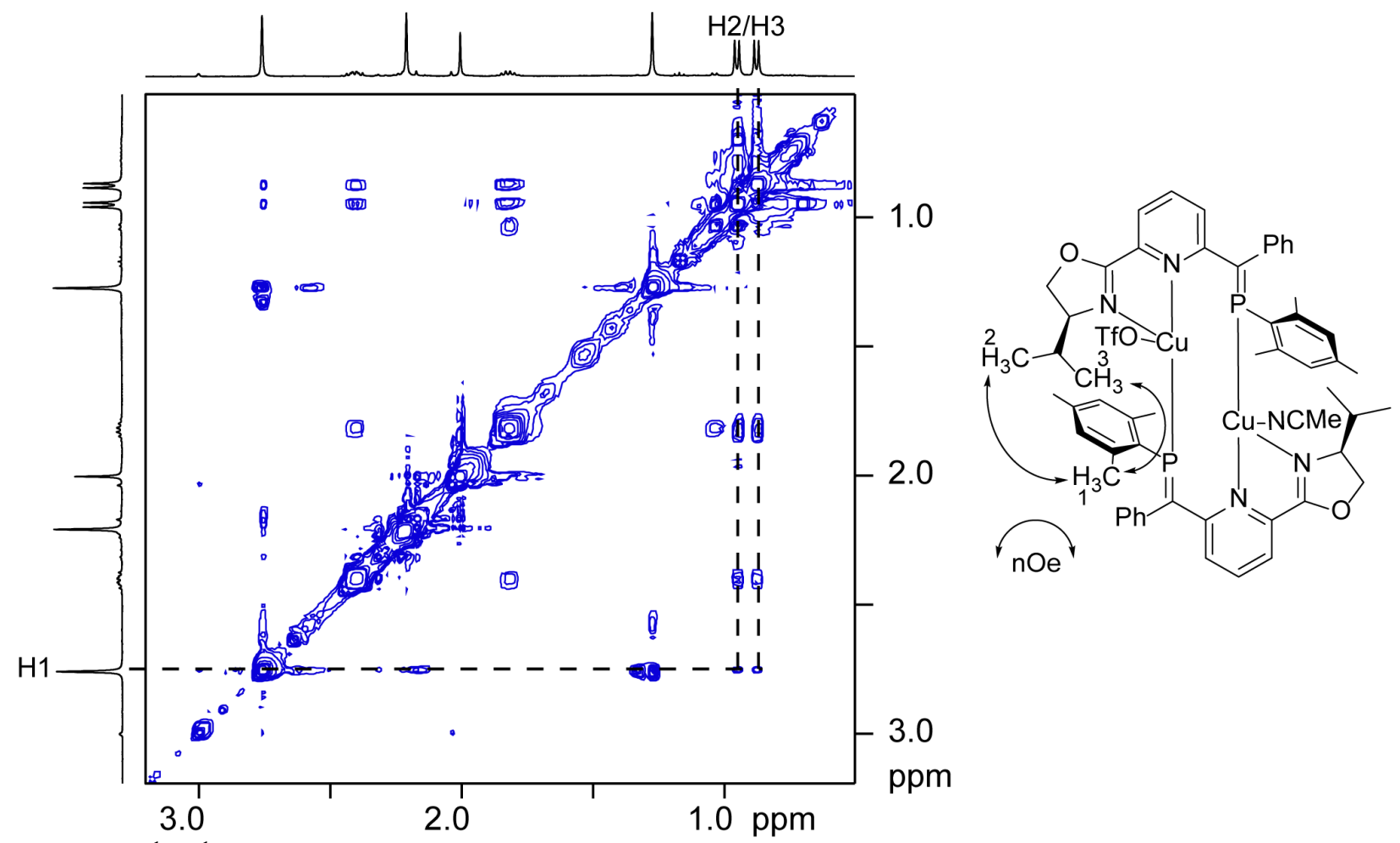

Figure S3 ${ }^{1} \mathrm{H}-{ }^{1} \mathrm{H}$ NOESY NMR spectrum $(400 \mathrm{MHz}, 298 \mathrm{~K})$ in $\mathrm{CDCl}_{3}$ of complex 7. 


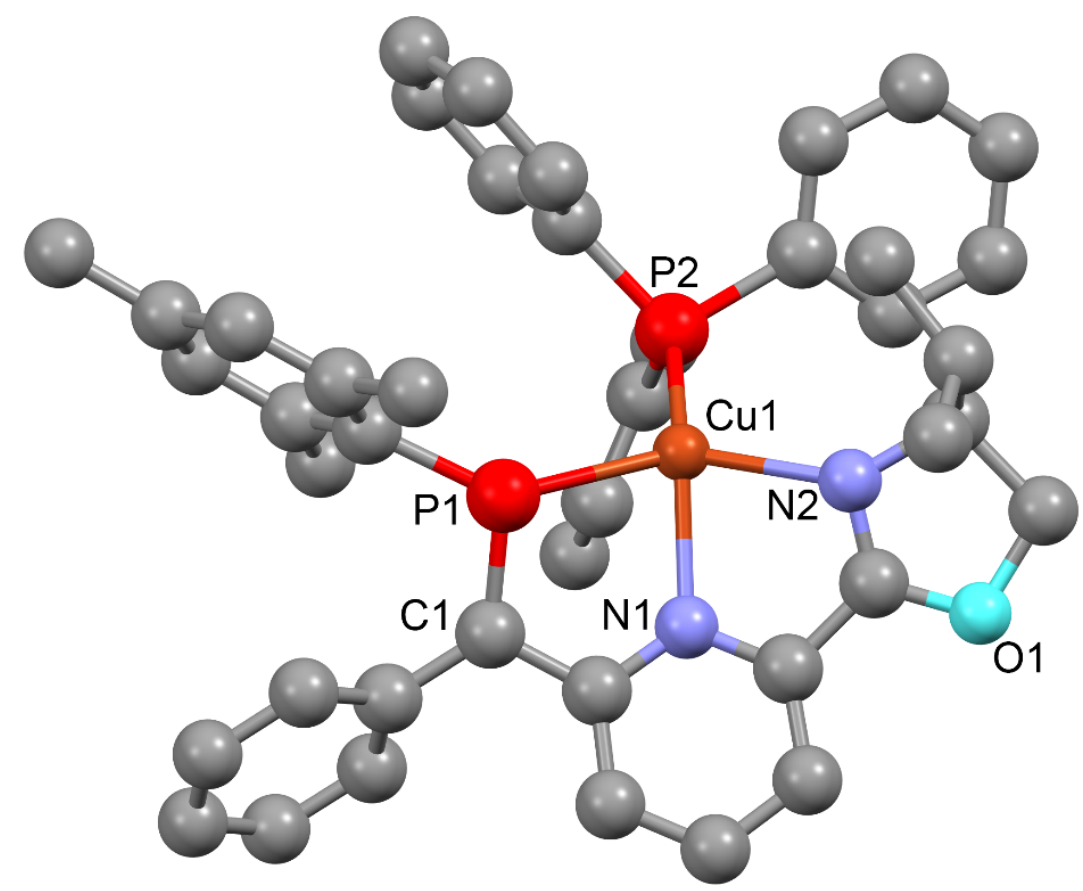

Figure S4. Optimized structure of 8a. Selected bond distances $(\AA)$ and angles $\left(^{\circ}\right)$ : $\mathrm{P}(1)-\mathrm{C}(1)$ 1.725, $\mathrm{P}(1)-\mathrm{Cu}(1)$ 2.414, $\mathrm{N}(1)-\mathrm{Cu}(1)$ 2.126, $\mathrm{N}(2)-\mathrm{Cu}(1)$ 2.152, $\mathrm{P}(2)-\mathrm{Cu}(1)$ 2.319, $\mathrm{C}(1)-\mathrm{P}(1)-\mathrm{Cu}(1)$ 101.47, $\mathrm{P}(1)-\mathrm{Cu}(1)-\mathrm{N}(1) 76.70, \mathrm{~N}(1)-\mathrm{Cu}(1)-\mathrm{N}(2) 77.66$.

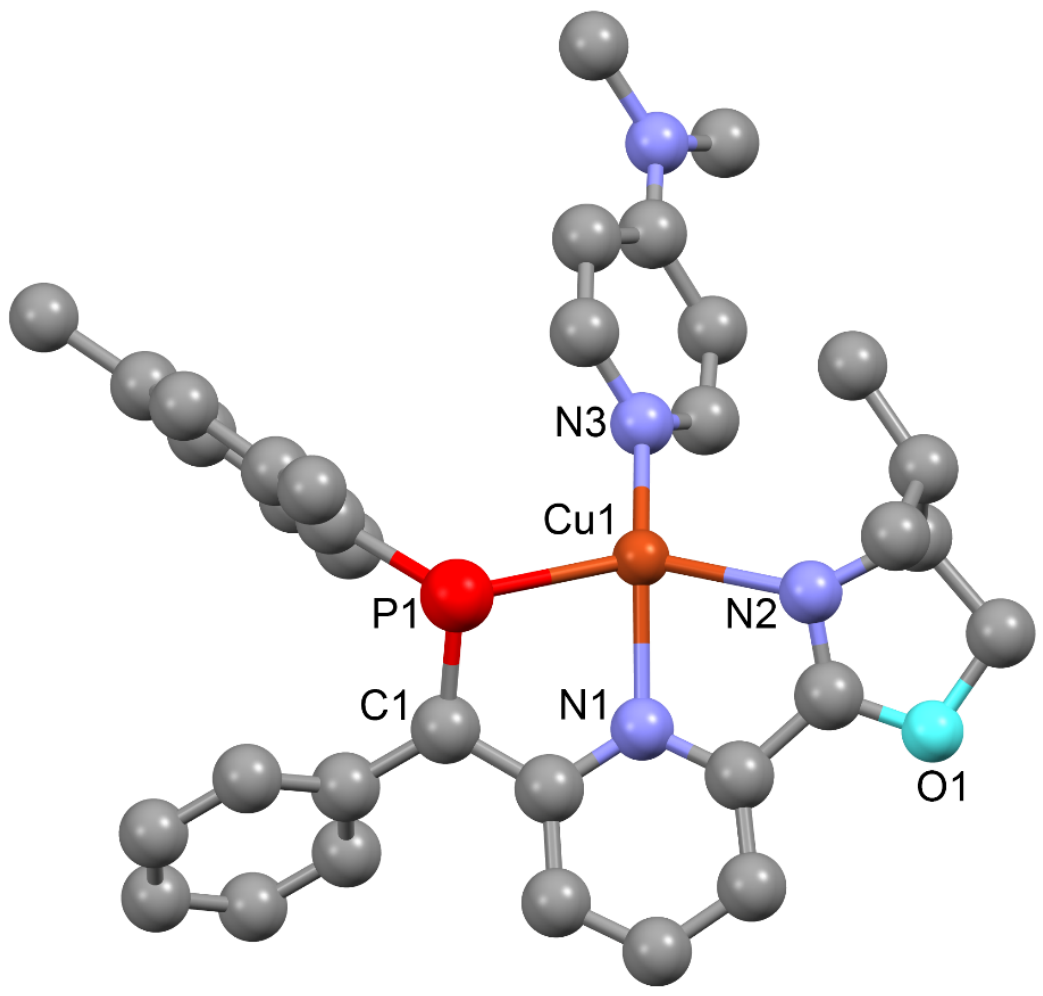

Figure S5. Optimized structure of 8d. Selected bond distances $(\AA)$ and angles $\left(^{\circ}\right)$ : $\mathrm{P}(1)-\mathrm{C}(1)$ 1.730, $\mathrm{P}(1)-\mathrm{Cu}(1)$ 2.387, $\mathrm{N}(1)-\mathrm{Cu}(1)$ 2.084, $\mathrm{N}(2)-\mathrm{Cu}(1)$ 2.183, $\mathrm{N}(3)-\mathrm{Cu}(1)$ 2.016, $\mathrm{C}(1)-\mathrm{P}(1)-\mathrm{Cu}(1)$ 99.55, $\mathrm{P}(1)-\mathrm{Cu}(1)-\mathrm{N}(1) 78.70, \mathrm{~N}(1)-\mathrm{Cu}(1)-\mathrm{N}(2) 78.11$. 


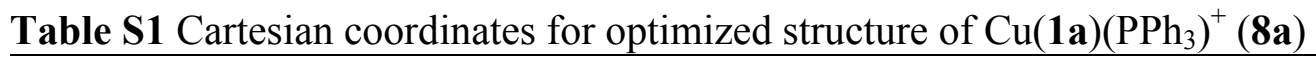

\begin{tabular}{|c|c|c|c|}
\hline $\mathrm{Cu}$ & -0.784651 & -0.623365 & -0.378075 \\
\hline $\mathrm{P}$ & 1.580477 & -0.618074 & -0.862063 \\
\hline $\mathrm{P}$ & -1.516136 & 1.317378 & 0.660065 \\
\hline $\mathrm{O}$ & -3.434881 & -3.808598 & -0.332092 \\
\hline $\mathrm{N}$ & -2.449178 & -1.871306 & -0.929158 \\
\hline $\mathrm{N}$ & -0.147804 & -2.544437 & 0.27366 \\
\hline $\mathrm{C}$ & 3.518288 & -2.029627 & 0.725982 \\
\hline $\mathrm{C}$ & 1.14053 & -2.882815 & 0.478808 \\
\hline $\mathrm{C}$ & -0.398308 & 0.412249 & 3.06622 \\
\hline $\mathrm{H}$ & 0.134419 & -0.288184 & 2.435117 \\
\hline $\mathrm{C}$ & 3.409335 & 1.476229 & -0.138419 \\
\hline $\mathrm{C}$ & -1.292334 & 1.31976 & 2.493428 \\
\hline $\mathrm{C}$ & 3.71596 & -2.301231 & 2.09019 \\
\hline $\mathrm{H}$ & 2.86069 & -2.375526 & 2.751079 \\
\hline $\mathrm{C}$ & -0.847803 & -4.819565 & 0.540011 \\
\hline $\mathrm{H}$ & -1.645268 & -5.548499 & 0.514 \\
\hline $\mathrm{C}$ & -0.352988 & 3.92763 & 0.844695 \\
\hline $\mathrm{H}$ & -0.534297 & 3.888648 & 1.910027 \\
\hline $\mathrm{C}$ & 5.923303 & -2.099752 & 0.41807 \\
\hline $\mathrm{H}$ & 6.780328 & -2.032735 & -0.240236 \\
\hline $\mathrm{C}$ & 2.150709 & -1.852884 & 0.199273 \\
\hline $\mathrm{C}$ & 4.645442 & -1.934625 & -0.100647 \\
\hline $\mathrm{H}$ & 4.517606 & -1.745964 & -1.157642 \\
\hline $\mathrm{C}$ & 0.238878 & 5.061199 & 0.296363 \\
\hline $\mathrm{H}$ & 0.50533 & 5.889739 & 0.940812 \\
\hline $\mathrm{C}$ & -5.604645 & 0.994413 & 0.98911 \\
\hline $\mathrm{H}$ & -6.292937 & 0.343167 & 1.514057 \\
\hline $\mathrm{C}$ & 4.784909 & 2.619974 & -1.799827 \\
\hline $\mathrm{C}$ & 4.994767 & -2.455946 & 2.608644 \\
\hline $\mathrm{H}$ & 5.125824 & -2.655006 & 3.665045 \\
\hline $\mathrm{C}$ & -2.361432 & -3.009246 & -0.346121 \\
\hline $\mathrm{C}$ & -0.698337 & 2.849062 & 0.027222 \\
\hline $\mathrm{C}$ & -4.235287 & 0.800585 & 1.121367 \\
\hline $\mathrm{H}$ & -3.870932 & -0.001643 & 1.75251 \\
\hline $\mathrm{C}$ & -1.094294 & -3.494175 & 0.218702 \\
\hline $\mathrm{C}$ & -3.826106 & 2.682853 & -0.32571 \\
\hline $\mathrm{H}$ & -3.146923 & 3.355922 & -0.831609 \\
\hline $\mathrm{C}$ & 2.887502 & 0.642347 & -1.148826 \\
\hline $\mathrm{C}$ & 0.150945 & 4.060766 & -1.890656 \\
\hline $\mathrm{H}$ & 0.348996 & 4.105797 & -2.954188 \\
\hline $\mathrm{C}$ & 3.292388 & 0.823502 & -2.492221 \\
\hline $\mathrm{C}$ & 4.349916 & 2.443518 & -0.489725 \\
\hline $\mathrm{H}$ & 4.752008 & 3.081592 & 0.289514 \\
\hline $\mathrm{C}$ & -0.428233 & 2.921923 & -1.3436 \\
\hline $\mathrm{H}$ & -0.671108 & 2.086225 & -1.989579 \\
\hline $\mathrm{C}$ & -4.502045 & -3.054361 & -0.990281 \\
\hline $\mathrm{H}$ & -5.26391 & -2.849462 & -0.239678 \\
\hline $\mathrm{H}$ & -4.91943 & -3.686856 & -1.7709 \\
\hline $\mathrm{C}$ & -3.331396 & 1.650179 & 0.471712 \\
\hline $\mathrm{C}$ & 6.103868 & -2.356464 & 1.773577 \\
\hline $\mathrm{H}$ & 7.100964 & -2.483477 & 2.175834 \\
\hline
\end{tabular}




\begin{tabular}{|c|c|c|c|}
\hline $\mathrm{C}$ & 3.002088 & 1.365978 & 1.30748 \\
\hline $\mathrm{H}$ & 1.922782 & 1.267104 & 1.422119 \\
\hline $\mathrm{H}$ & 3.313106 & 2.251934 & 1.860308 \\
\hline $\mathrm{H}$ & 3.46405 & 0.499293 & 1.784992 \\
\hline $\mathrm{C}$ & 4.240419 & 1.798223 & -2.785768 \\
\hline $\mathrm{H}$ & 4.563356 & 1.919334 & -3.814088 \\
\hline $\mathrm{C}$ & 0.486168 & 5.133092 & -1.070265 \\
\hline $\mathrm{H}$ & 0.943421 & 6.018595 & -1.493611 \\
\hline $\mathrm{C}$ & 1.463747 & -4.214532 & 0.803931 \\
\hline $\mathrm{H}$ & 2.495128 & -4.48869 & 0.970169 \\
\hline $\mathrm{C}$ & 0.463083 & -5.168433 & 0.865412 \\
\hline $\mathrm{H}$ & 0.705744 & -6.192457 & 1.118814 \\
\hline $\mathrm{C}$ & -1.998469 & 2.197256 & 3.32457 \\
\hline $\mathrm{H}$ & -2.717642 & 2.887683 & 2.902628 \\
\hline $\mathrm{C}$ & -6.091572 & 2.027728 & 0.191472 \\
\hline $\mathrm{H}$ & -7.15874 & 2.180197 & 0.090411 \\
\hline $\mathrm{C}$ & 2.759648 & -0.032274 & -3.618703 \\
\hline $\mathrm{H}$ & 3.05694 & -1.079161 & -3.513992 \\
\hline $\mathrm{H}$ & 3.137893 & 0.320789 & -4.57781 \\
\hline $\mathrm{H}$ & 1.667777 & -0.015628 & -3.666508 \\
\hline $\mathrm{C}$ & -3.804334 & -1.774349 & -1.517972 \\
\hline $\mathrm{H}$ & -4.290611 & -0.885409 & -1.114901 \\
\hline $\mathrm{C}$ & -0.197519 & 0.393496 & 4.443092 \\
\hline $\mathrm{H}$ & 0.496751 & -0.315127 & 4.877243 \\
\hline $\mathrm{C}$ & -0.893766 & 1.276634 & 5.260356 \\
\hline $\mathrm{H}$ & -0.741255 & 1.2598 & 6.332251 \\
\hline $\mathrm{C}$ & 5.788118 & 3.686274 & -2.150795 \\
\hline $\mathrm{H}$ & 5.288429 & 4.57311 & -2.551651 \\
\hline $\mathrm{H}$ & 6.488632 & 3.338508 & -2.911889 \\
\hline $\mathrm{H}$ & 6.360809 & 3.998617 & -1.277195 \\
\hline $\mathrm{C}$ & -3.25042 & -0.277988 & -3.492816 \\
\hline $\mathrm{H}$ & -3.821445 & 0.53349 & -3.036733 \\
\hline $\mathrm{H}$ & -3.318435 & -0.168024 & -4.576531 \\
\hline $\mathrm{H}$ & -2.201544 & -0.15511 & -3.213031 \\
\hline $\mathrm{C}$ & -3.77495 & -1.649262 & -3.057196 \\
\hline $\mathrm{H}$ & -4.825299 & -1.717331 & -3.365578 \\
\hline $\mathrm{C}$ & -3.002418 & -2.781449 & -3.74152 \\
\hline $\mathrm{H}$ & -1.937502 & -2.728262 & -3.505484 \\
\hline $\mathrm{H}$ & -3.105104 & -2.705665 & -4.825154 \\
\hline $\mathrm{H}$ & -3.363262 & -3.77141 & -3.453217 \\
\hline $\mathrm{C}$ & -5.20017 & 2.867894 & -0.464513 \\
\hline $\mathrm{H}$ & -5.569926 & 3.67862 & -1.079976 \\
\hline $\mathrm{C}$ & -1.796467 & 2.176139 & 4.699261 \\
\hline $\mathrm{H}$ & -2.348075 & 2.858935 & 5.333422 \\
\hline
\end{tabular}


Table S2 Cartesian coordinates for optimized structure of $\mathrm{Cu}(\mathbf{1 a})(4-\mathrm{DMAP})^{+}(\mathbf{8 d})$

\begin{tabular}{|c|c|c|c|}
\hline $\mathrm{Cu}$ & 1.020498 & -0.558459 & 0.131245 \\
\hline $\mathrm{P}$ & -1.2592 & -0.467995 & 0.833389 \\
\hline $\mathrm{O}$ & 3.626781 & -3.751794 & -0.513164 \\
\hline $\mathrm{N}$ & 2.812978 & -1.796928 & 0.262392 \\
\hline $\mathrm{N}$ & 0.315831 & -2.40498 & -0.530659 \\
\hline $\mathrm{C}$ & -3.322847 & -1.697416 & -0.751305 \\
\hline $\mathrm{C}$ & -1.001478 & -2.704101 & -0.584263 \\
\hline $\mathrm{C}$ & -2.69864 & 1.825104 & -0.094242 \\
\hline $\mathrm{C}$ & -3.567268 & -1.981339 & -2.105978 \\
\hline $\mathrm{H}$ & -2.733071 & -2.145125 & -2.777335 \\
\hline $\mathrm{C}$ & 0.901776 & -4.704247 & -0.878846 \\
\hline $\mathrm{H}$ & 1.673887 & -5.455397 & -0.966622 \\
\hline $\mathrm{C}$ & -5.720086 & -1.536599 & -0.408978 \\
\hline $\mathrm{H}$ & -6.557014 & -1.375697 & 0.258969 \\
\hline $\mathrm{C}$ & -1.939255 & -1.633332 & -0.248607 \\
\hline $\mathrm{C}$ & -4.424757 & -1.478722 & 0.087668 \\
\hline $\mathrm{H}$ & -4.263305 & -1.283151 & 1.139063 \\
\hline $\mathrm{C}$ & -3.943559 & 3.287303 & 1.414508 \\
\hline $\mathrm{C}$ & -4.863182 & -2.031186 & -2.602072 \\
\hline $\mathrm{H}$ & -5.028675 & -2.242748 & -3.651184 \\
\hline $\mathrm{C}$ & 2.590863 & -2.934868 & -0.281167 \\
\hline $\mathrm{C}$ & 1.231521 & -3.389212 & -0.602267 \\
\hline $\mathrm{C}$ & -2.385833 & 0.977513 & 0.991351 \\
\hline $\mathrm{C}$ & -2.825619 & 1.30964 & 2.293363 \\
\hline $\mathrm{C}$ & -3.472396 & 2.959548 & 0.145478 \\
\hline $\mathrm{H}$ & -3.710604 & 3.610149 & -0.689108 \\
\hline $\mathrm{C}$ & 4.816152 & -3.027374 & -0.068046 \\
\hline $\mathrm{H}$ & 5.454025 & -2.88406 & -0.938376 \\
\hline $\mathrm{H}$ & 5.329325 & -3.651543 & 0.660909 \\
\hline $\mathrm{C}$ & -5.944994 & -1.809546 & -1.75499 \\
\hline $\mathrm{H}$ & -6.955944 & -1.85472 & -2.13988 \\
\hline $\mathrm{C}$ & -2.234883 & 1.55952 & -1.504562 \\
\hline $\mathrm{H}$ & -1.191925 & 1.242372 & -1.541834 \\
\hline $\mathrm{H}$ & -2.336867 & 2.457996 & -2.113167 \\
\hline $\mathrm{H}$ & -2.830787 & 0.774221 & -1.974576 \\
\hline $\mathrm{C}$ & -3.601262 & 2.450827 & 2.475554 \\
\hline $\mathrm{H}$ & -3.946766 & 2.693904 & 3.474409 \\
\hline $\mathrm{C}$ & -1.403376 & -4.028175 & -0.851856 \\
\hline $\mathrm{H}$ & -2.456944 & -4.263995 & -0.892614 \\
\hline $\mathrm{C}$ & -0.452299 & -5.015968 & -1.021852 \\
\hline $\mathrm{H}$ & -0.756679 & -6.034032 & -1.227877 \\
\hline $\mathrm{C}$ & -2.511797 & 0.445611 & 3.49265 \\
\hline $\mathrm{H}$ & -3.024233 & -0.519063 & 3.442954 \\
\hline $\mathrm{H}$ & -2.828368 & 0.936546 & 4.412644 \\
\hline $\mathrm{H}$ & -1.443559 & 0.232111 & 3.579191 \\
\hline $\mathrm{C}$ & 4.266424 & -1.700354 & 0.52064 \\
\hline $\mathrm{H}$ & 4.655711 & -0.847417 & -0.040816 \\
\hline $\mathrm{C}$ & -4.813172 & 4.496811 & 1.631956 \\
\hline $\mathrm{H}$ & -4.660944 & 4.925754 & 2.623375 \\
\hline $\mathrm{H}$ & -5.871165 & 4.229962 & 1.551882 \\
\hline $\mathrm{H}$ & -4.615851 & 5.270577 & 0.888989 \\
\hline
\end{tabular}




\begin{tabular}{llll}
$\mathrm{C}$ & 4.218724 & -0.059783 & 2.457281 \\
$\mathrm{H}$ & 4.687943 & 0.699437 & 1.827172 \\
$\mathrm{H}$ & 4.53892 & 0.116246 & 3.485598 \\
$\mathrm{H}$ & 3.137625 & 0.088267 & 2.41611 \\
$\mathrm{C}$ & 4.598749 & -1.474873 & 2.011463 \\
$\mathrm{H}$ & 5.690137 & -1.56267 & 2.077388 \\
$\mathrm{C}$ & 3.978587 & -2.531067 & 2.931569 \\
$\mathrm{H}$ & 2.888999 & -2.460983 & 2.930086 \\
$\mathrm{H}$ & 4.31949 & -2.382366 & 3.957274 \\
$\mathrm{H}$ & 4.251394 & -3.549865 & 2.647189 \\
$\mathrm{C}$ & 1.329712 & 2.389737 & 0.322383 \\
$\mathrm{C}$ & 1.762816 & 3.656356 & 0.006595 \\
$\mathrm{C}$ & 2.550094 & 3.869673 & -1.151175 \\
$\mathrm{C}$ & 2.83977 & 2.710761 & -1.913338 \\
$\mathrm{C}$ & 2.361102 & 1.485707 & -1.510952 \\
$\mathrm{~N}$ & 1.610692 & 1.292043 & -0.407609 \\
$\mathrm{H}$ & 2.583096 & 0.603639 & -2.098345 \\
$\mathrm{H}$ & 3.430641 & 2.762697 & -2.81463 \\
$\mathrm{H}$ & 0.72029 & 2.23534 & 1.203413 \\
$\mathrm{H}$ & 1.482206 & 4.4719 & 0.654906 \\
$\mathrm{~N}$ & 2.99538 & 5.098937 & -1.506864 \\
$\mathrm{C}$ & 2.674661 & 6.263649 & -0.68756 \\
$\mathrm{H}$ & 3.060456 & 6.154926 & 0.329667 \\
$\mathrm{H}$ & 1.59547 & 6.433642 & -0.636391 \\
$\mathrm{C}$ & 3.790848 & 5.273795 & -2.717959 \\
$\mathrm{H}$ & 3.241237 & 4.954569 & -3.607782 \\
$\mathrm{H}$ & 4.729352 & 4.714544 & -2.666508 \\
$\mathrm{H}$ & 3.133735 & 7.143611 & -1.128449 \\
$\mathrm{H}$ & 4.033311 & 6.326154 & -2.832838 \\
\hline
\end{tabular}


Table S3 Computed Low Energy Absorptions for 8a

\begin{tabular}{|c|c|c|c|}
\hline state no. & $\begin{array}{l}\text { calculated energy } \\
\text { /eV }\end{array}$ & $f$ & constitution $^{a, b}$ \\
\hline 1 & 2.3412 & 0.0763 & $\begin{array}{l}197-198(66.2 \%), 196-198(15 \%), 194-198(12.3 \%), \\
193-198(11.3 \%)\end{array}$ \\
\hline 2 & 2.7614 & 0.0166 & $197-199(65.7 \%), 193-199(10.8 \%)$ \\
\hline 3 & 2.9232 & 0.0707 & $196-198(51.8 \%), 195-198(24.7 \%), 194-198(22.1 \%)$ \\
\hline 4 & 2.9901 & 0.0318 & $195-198(64.1 \%)$ \\
\hline 5 & 3.0106 & 0.0732 & 194-198 (55.4\%), 193-198 (18.7\%), 195-198 (12.9\%) \\
\hline 6 & 3.0530 & 0.0205 & $193-198(59.1 \%), 189-198(17.3 \%), 183-198(12.3 \%)$ \\
\hline
\end{tabular}

Table S4 Computed Low Energy Absorptions for 8d

\begin{tabular}{|c|c|c|c|}
\hline state no. & $\begin{array}{l}\text { calculated energy } \\
\text { /eV }\end{array}$ & $f$ & constitution $^{a, b}$ \\
\hline 1 & 2.2523 & 0.0680 & $161-162(67.1 \%)$ \\
\hline 2 & 2.5263 & 0.0209 & $161-163(68.7 \%)$ \\
\hline 3 & 2.8379 & 0.0236 & $\begin{array}{l}160-162(47.1 \%), 159-162(38.8 \%), 154-162(18.0 \%) \\
157-162(16.0 \%)\end{array}$ \\
\hline 4 & 2.9758 & 0.0774 & $159-162(64.1 \%), 151-162(14.8 \%), 161-162(12.4 \%)$ \\
\hline 5 & 3.1272 & 0.0024 & $158-162(62.0 \%), 159-162(14.9 \%)$ \\
\hline 6 & 3.1602 & 0.02558 & $157-162(59.1 \%), 158-162(28.1 \%), 155-162(22.0 \%)$ \\
\hline
\end{tabular}


Table S5 X-ray Data Collection and Refinement Details

\begin{tabular}{|c|c|c|c|c|}
\hline & $3 \mathbf{a}$ & $Z-1 \mathbf{a}$ & 7 & $8 \mathbf{a} \cdot\left(\mathrm{C}_{4} \mathrm{H}_{8} \mathrm{O}\right)_{3}$ \\
\hline formula & $\mathrm{C}_{18} \mathrm{H}_{18} \mathrm{~N}_{2} \mathrm{O}_{2}$ & $\mathrm{C}_{27} \mathrm{H}_{29} \mathrm{~N}_{2} \mathrm{OP}$ & $\begin{array}{c}\mathrm{C}_{57} \mathrm{H}_{61} \mathrm{Cu}_{2} \mathrm{~F}_{3} \mathrm{~N}_{5} \mathrm{O}_{5} \\
\left.\mathrm{P}_{2} \mathrm{~S}\right]\left[\mathrm{CF}_{3} \mathrm{SO}_{3}\right]\end{array}$ & $\begin{array}{c}\left.\mathrm{C}_{45} \mathrm{H}_{44} \mathrm{CuN}_{2} \mathrm{OP}_{2}\right] \\
{\left[\mathrm{CF}_{3} \mathrm{SO}_{3}\right]} \\
\cdot 3\left(\mathrm{C}_{4} \mathrm{H}_{8} \mathrm{O}\right)\end{array}$ \\
\hline fw & 294.34 & 428.49 & 1323.01 & 1115.65 \\
\hline cryst syst & monoclinic & monoclinic & triclinic & orthorhombic \\
\hline space group & $P 2_{1}$ & $P 2_{1}$ & $P 1$ & $P 2{ }_{1} 2_{1} 2_{1}$ \\
\hline colour & colourless & yellow & orange & red \\
\hline$a(\AA)$ & $19.628(1)$ & $8.6564(8)$ & $11.399(1)$ & $12.0076(5)$ \\
\hline$b(\AA)$ & $6.5495(4)$ & $6.4176(6)$ & $11.762(1)$ & $15.2895(6)$ \\
\hline$c(\AA)$ & $12.9494(9)$ & $21.018(2)$ & $12.253(1)$ & $29.640(1)$ \\
\hline$\alpha(\operatorname{deg})$ & 90 & 90 & $92.416(2)$ & 90 \\
\hline$\beta(\operatorname{deg})$ & $111.225(5)$ & $99.396(2)$ & $107.396(2)$ & 90 \\
\hline$\gamma(\operatorname{deg})$ & 90 & 90 & $104.977(2)$ & 90 \\
\hline$V\left(\AA^{3}\right)$ & $1551.8(2)$ & $1151.9(2)$ & $1501.8(3)$ & $5441.5(4)$ \\
\hline $\mathrm{T}(\mathrm{K})$ & $90(2)$ & $90(2)$ & $90(2)$ & $90(2)$ \\
\hline $\mathrm{Z}$ & 4 & 2 & 1 & 4 \\
\hline$\mu(\operatorname{Mo~K} \alpha)\left(\mathrm{mm}^{-1}\right)$ & 0.083 & 0.141 & 0.907 & 0.563 \\
\hline cryst size $(\mathrm{mm})$ & $0.25 \times 0.14 \times 0.13$ & $0.34 \times 0.12 \times 0.10$ & $0.28 \times 0.12 \times 0.10$ & $0.15 \times 0.12 \times 0.04$ \\
\hline $\mathrm{D}_{\text {calcd. }}\left(\mathrm{Mg} \mathrm{m}^{-3}\right)$ & 1.260 & 1.235 & 1.463 & 1.362 \\
\hline $2 \theta(\max )\left(^{\circ}\right)$ & 60.1 & 60.2 & 60.2 & 60.2 \\
\hline no. of reflns & 16885 & 13878 & 17750 & 15991 \\
\hline no. of unique data & 7438 & 6099 & 8875 & 8750 \\
\hline$R$ (int) & 0.0396 & 0.0436 & 0.0400 & 0.0504 \\
\hline refln/param ratio & 22.5 & 22.5 & 25.9 & 23.4 \\
\hline $\mathrm{R}_{1}[\mathrm{I}>2 \sigma(\mathrm{I})]^{a}$ & 0.0269 & 0.0332 & 0.0264 & 0.0328 \\
\hline $\mathrm{wR}_{2}\left[\right.$ all data] ${ }^{b}$ & 0.1035 & 0.0959 & 0.0977 & 0.0735 \\
\hline GOF & 1.047 & 1.014 & 1.043 & 0.947 \\
\hline $\mathrm{CCDC}$ & 1469496 & 1469495 & 1469497 & 1469498 \\
\hline
\end{tabular}

${ }^{a} R_{1}=\Sigma\left\|F_{o}|-| F_{c}\right\| / \Sigma\left|F_{o}\right|{ }^{b} \mathrm{w} R_{2}\left(F^{2}[\right.$ all data $\left.]\right)=\left\{\Sigma\left[w\left({F_{o}}^{2}-F_{c}{ }^{2}\right)^{2}\right] / \Sigma\left[w\left(F_{o}{ }^{2}\right)^{2}\right]\right\}^{1 / 2}$ 